\title{
Development of a Scale to Evaluate the Depressive State among Elderly Patients in General Wards
}

\author{
Tetsuko Takaoka' ${ }^{1}$, Ruriko Kidachi ${ }^{2}$ \\ ${ }^{1}$ Department of Development and Aging, Hirosaki University Graduate School of Health Sciences, Hirosaki, Japan \\ ${ }^{2}$ Department of Nursing Science, Hirosaki University Graduate School of Health Sciences, Hirosaki, Japan \\ Email: tetsumo@do-bunkyodai.ac.jp
}

How to cite this paper: Takaoka, T. and Kidachi, R. (2017) Development of a Scale to Evaluate the Depressive State among Elderly Patients in General Wards. Open Journal of Nursing, 7, 495-512. https://doi.org/10.4236/ojn.2017.74039

Received: March 4, 2017

Accepted: April 27, 2017

Published: April 30, 2017

Copyright @ 2017 by authors and Scientific Research Publishing Inc. This work is licensed under the Creative Commons Attribution International License (CC BY 4.0).

http://creativecommons.org/licenses/by/4.0/ (c) (i) Open Access

\begin{abstract}
This study aims to develop a scale to evaluate depressive states among elderly patients in general wards, and verify the reliability and validity of the scale. Based on the results of interviews with nurses, we developed a draft of a scale comprised of 48 questions (NDE48). The Geriatric Depression Scale short version (GDS15), and the Zung Self-rating Depression Scale (SDS) were administered to 54 elderly patients. Two groups of nurses completed the NDE48 on different days. After performing an explanatory factor analysis, the NDE48 was simplified to a 16 question item scale (NDE16), comprising three factors. The correlation coefficient between GDS15 and NDE16 was 0.41 ( $p=$ $0.00191)$, and between SDS and NDE16 was $0.30(p=0.02633)$. Alpha coefficient of the total score of NDE16 was 0.88 . For inter-rater reliability, the correlation coefficient was $0.57(p=0.00005)$. The findings suggest that the NDE16 will be highly useful when nurses evaluate depressive states among elderly inpatients.
\end{abstract}

\section{Keywords}

Depressive States, Elderly Patients, General Ward, Non-Self-Rating Scale, Nurses

\section{Introduction}

As the ratio of the aged population (26.7\% in 2016) in Japan [1] has increased and the life expectancy also continues to lengthen, the ratio of elderly people receiving hospital treatment (2840 inpatients/100,000 elderly people, 10,637 outpatients/100,000 elderly people) is expected to become higher. With this background the Japanese government has promoted measures to shorten the length 
of hospital stays to curb medical costs, and medical institutions have also aimed to shorten the length of stays by using critical path analysis [2] to improve care efficiency and quality. However, compared to that of Australia (4.7 days) and Italy (6.8 days), the 17.2 days in Japan is long (OECD) [3]. This suggests that there are more elderly inpatients in general in Japan than in other countries. In Japan, the term "general wards" excludes wards for psychiatric medicine, infectious diseases, tuberculosis, and recuperation in clinics or hospitals.

Further, the incidence of depressive disorders among elderly patients in general wards amounted $22 \%$ to $33 \%$ of those admitted, a higher incidence than in the general community where the incidence is $5 \%$ to $15 \%$ [4]. This could suggest that the elderly experience depressive states triggered by the hospital admission, or it could offer an opportunity to determine depressive states among elderly inpatients. However, there is a risk to overlook the changes in the state of mind of elderly inpatients because attention to inpatients is primarily paid to improving the main complaint that led to the hospital admission. To verify this assumption, we first reviewed literature related to depression among the elderly related to nursing sciences in Japan, and determined that it is necessary to understand the actual conditions of the depressive states and depression, and improve nursing intervention for early detection and prevention, as well as to advance research into depressive states targeting elderly inpatients, due to the high incidence of depressive disorders [5]. Therefore, it is of significant importance to screen for early stage depressive states among elderly inpatients in Japan.

The primary feature of depressive states among the elderly is that they show many somatic symptoms (indefinite, no certain diagnosis possible complaints) [6] as well as practice thought suppression and inhibition [6], and depression among the elderly seem to be accompanied by complaints of prolonged poor health, hypochondriac symptoms, anxiety, feelings of frustration, and delusions [7]. In this way, the elderly show a wider range of conditions than those of adolescents or middle aged patients [8], and we consider that this difference makes it difficult to identify early stage depressive states among the elderly, and that it is necessary to identify factors related to depression among the elderly for early detection. Items extracted as factors related to depressive states among the elderly include physical factors that include physical states and illnesses [9]-[14], psychological factors [15] [16], and social factors [17] from studies conducted by researchers in Japan and other countries.

The Geriatric Depression Scale short version (GDS15) [18] and the Zung Self-rating Depression Scale (SDS) [19] are known as self-administered scales to measure the depressive state among the elderly. Nakane et al. studied knowledge and understanding of (attitudes towards) psychiatric disorders among the general population of Japan and Australia, and reported that the Japanese had more negative attitudes towards patients suffering from depression [20]. This suggests that Japanese have more negative impressions of depression. For this reason, administering a self-rated depression scale to elderly patients at hospital admission may impose a psychological burden on these patients. Therefore, it would 
be preferable to have a scale that can be completed by attending nurses, to decrease the psychological burden on patients when depressive states are evaluated. The Checklist for Post Stroke Depression (CPSD) for stroke patients [21], the Delirium, Depression, Dementia Screening Tool (3DST) for elderly patients under palliative care [22], and the Nursing homes short depression inventory (NH-SDI) for elderly residents at nursing homes [23] are among scales that are completed by attending nurses. As described above, the incidence of depressive disorders among elderly patients in general wards is high, and there is a need to develop a scale exclusively for such elderly patients. However, our extensive research review found no previous studies related to such scales. Therefore, we believe it would be valuable to develop a scale to evaluate depressive states among elderly inpatients without disturbing the main object (illness) of the hospitalization of elderly patients and that the scale should minimize the burden on these patients.

Observation of hospital inpatients by nurses is also one way that would make it possible to detect depressive states among elderly inpatients, other than by using a set scale. Because nurses spend more time with patients than other health professionals attending patients, attending nurses may be able to evaluate depressive states as they develop over time, and not just at a single point in time, as nurses actually do with the CPSD [21], the 3DST [22], or the NH-SDI [23] described above. Benner classified the length of nursing experience into five stages, from novice to expert, and has reported that novice nurses regard everything as important information, but as their experience becomes more extensive, they will become better able to see situations as a complete whole [24]. This points to the situation that nurse assessment abilities vary with the length of nursing experience, and that as a result it is necessary to establish standard criteria to ensure that also novice nurses can provide a valid assessment of the state investigated. In this study we aim to develop a scale which nurses will be able to apply without complications in evaluating depressive states among elderly patients in general wards both in and outside Japan. It is expected that such a scale will facilitate an early detection of underlying depressive states among elderly inpatients, lead to opportunities for inpatients to receive appropriate treatment from physicians, and contribute to improvements in the quality of life (QOL) of those where depression is identified, including assisting in the prevention of suicides. Further, we believe that carrying out this study is meaningful because the findings will also contribute to achieving recovery from the main reasons for the hospitalization of elderly patients, as well as that this will contribute to decreases in medical outlays.

\section{Objectives}

The objectives of this study are to develop a scale to screen the depressive state among elderly patients in general wards.

\section{Definition of Terms}

As previously described, depressive states among the elderly are accompanied by 
thought suppression and inhibition [6]. For this reason, Takaoka et al. [5] estimated that depressive states entail a high risk to pose obstacles in daily life activities. Therefore, we defined the term depressive state in this study The term depressive state in this study is defined as the level of depression where persons are depressed for a specific reason and are unable to cope with this by themselves, and which poses obstacles in daily life activities, including loss of appetite and insomnia, resulting in the need for nursing intervention. This is because the purpose of the scale is to measure the depressive state rather than to diagnose, and as we emphasize the importance of performing the measuring from the viewpoint of the attending nurses.

\section{Methods}

\subsection{Creation of a Draft Scale to Evaluate the Depressive State among Elderly Patients in General Wards}

\subsubsection{Development of the Question Items}

As this study emphasizes the importance of measuring the depressive states from the viewpoint of attending nurses, we decided to determine question items based on the daily observations by nurses. A semi-structured interview as one "criterion for nurse based evaluations of depressive states among elderly patients" was conducted in August, 2014. The interview was based on cases of nursing interventions that were conducted when elderly inpatients became depressed, and cases arising from follow-up observation. All the interviewees were female between 30 and 60 years of age. The nurse licenses held by 20 of the interviewees were obtained at vocational nursing schools. The mean length of experience as a nurse was $19.9 \pm 7.8$ years, the duration of working in the present ward $5.0 \pm 3.4$ years, and that of working in general wards $14.5 \pm 7.8$ years. The mean length of these interviews was $22.2 \pm 5.7$ minutes. The interviewees were 22 nurses with three years or longer clinical experience in general wards, who had been introduced to the researchers by the hospitals participating in this study. We studied the data obtained in the interviews, distinguished the information into codes with similar characteristics, and assigned names to represent the characteristics of the matter expressed by a code. We repeated this procedure and were able to categorize the codes into integrated groups; this finally yielded eleven categories detailed in 101 tertiary level codes (final codes) for use as query items.

With this procedure, it became clear that nurses evaluate depressive states among elderly inpatients based on the following eleven aspects (categories): \#1 $<<$ conditions requiring nursing intervention $>>, \# 2<<$ conditions with risks of committing suicide $>>$, $\# 3<<$ conditions showing lack of sufficient nutrition $>>$, $\# 4<<$ conditions showing lack of adequate rest $>>$, \#5 $<<$ conditions where physical functions are impaired $>>, \# 6<<$ conditions where the future livelihood cannot be imagined $>>, \# 7<<$ conditions motivating continued treatment not assured $>>$, \#8<<conditions involving negative feelings $>>$, \#9 $<<$ conditions specific to depressive characteristics in the elderly $>>, \# 10<<$ conditions due to the characteristics of being elderly $>>$, and $\# 11<<$ conditions due to hospitaliza- 
tion>>. Using the tertiary level codes that are components of the categories, specific question items were developed. Further, we simplified the expressions in the wordings to enable a determination regardless of the length of the nurse working experience. From the 101 codes, we extracted 48 codes based on the following exclusion criteria: "(1) Opposite results were identified”, “(2) Corresponds to all elderly", "(3) Number of segments extracted from the interviews was five or fewer", "(4) Seems difficult to evaluate”, “(5) Always performed for inpatients" and "Demographic information".

\subsubsection{Creation of the Scale}

To create a scale that is simple to answer, we employed a "Yes, No, or Unknown" style to the answers for each question, and a higher number of "Yes" answers suggests a higher probability of the presence of a depressive state.

\subsubsection{Evaluation of Content Validity}

The validity of the questions in terms of the relevance and simplicity and of the ability of the criteria to identify depressive states among the elderly was evaluated by three tests: with "comments from an expert panel consisting of a psychiatrist and gerontological nurses", with "a preliminary test conducted by two novice nurses and five nurses randomly selected from among the nurses participated in the semi-structured interview", and with "comparisons with previous studies related to depressive states among the elderly". Incorporating the modifications suggested by this validity check in the question wordings, the draft of the scale comprising 48 questions was complete.

\subsection{Evaluation of the Reliability and Validity of the Scale for Nurses to Evaluate Depressive States among Elderly Patients in General Wards}

\subsubsection{Instrument}

As the instrument, we used the final draft scale to evaluate depressive states among elderly patients in general wards comprised of 48 questions created in this study. The draft scale is termed the Nurse Administered Depression Scale for Elderly Inpatients (NDE48), and Table 1 shows the question items.

\subsubsection{Research Period}

Data were collected from February to December, 2015.

\subsubsection{Participants}

Participants surveyed were 65 year or older inpatients in general wards of the participating hospitals. Inclusion criteria were patients hospitalized for 2 to 7 days after the admission who were not diagnosed with dementia or depression and who consented to participate in the study. The nurses who were asked to complete a scale developed in this study were licensed nurses in charge of the ward where participants were hospitalized (attending nurses).

\subsubsection{Data Collection}

A researcher conducted the interviews with the participants using the Japanese 
Table 1. Questions in the scale to evaluate depressive states among elderly inpatients.

\begin{tabular}{|c|c|}
\hline No & Question \\
\hline 1 & Showing somatic symptoms \\
\hline 2 & Displaying worthlessness feeling \\
\hline 3 & Need for assistance to alleviate pain \\
\hline 4 & $\begin{array}{l}\text { Displaying physical strength weakening due to for being in bad condition or decrease } \\
\text { in exercises }\end{array}$ \\
\hline 5 & Appears dispirited \\
\hline 6 & Need some assistance in daily activities \\
\hline 7 & Presenting obstacles in daily life \\
\hline 8 & Daily activities are limited due to treatment \\
\hline 9 & $\begin{array}{l}\text { It is difficult for patient to try to avoid risks such as falls by him/herself. (added "such } \\
\text { as falls") }\end{array}$ \\
\hline 10 & Need to encourage patient to do something \\
\hline 11 & Stopped doing what he/she was able to \\
\hline 12 & $\begin{array}{l}\text { Unable to accept the present situation where activity is limited due to treatment or } \\
\text { obstacles to daily life }\end{array}$ \\
\hline 13 & $\begin{array}{l}\text { Need to make special arrangements for food types and/or eating style to increase the } \\
\text { ingested volume of food }\end{array}$ \\
\hline 14 & Patient is sometimes reluctant to eat or cannot eat even when encouraged. \\
\hline 15 & Patient is sometimes reluctant to drink water or not drinking even when encouraged. \\
\hline 16 & Complaining of being unable to sleep \\
\hline 17 & Showing negative attitudes toward encouragements \\
\hline 18 & Need to have a talk as a nursing aid \\
\hline 19 & Need encouragement to interact with others \\
\hline 20 & Reducing interactions with others \\
\hline 21 & Rejecting interactions with others \\
\hline 22 & Patient complains ("anxiety, dissatisfaction, and unspecified reasons" added). \\
\hline 23 & Patient makes many complaints ("frequent nurse calls, overly talkative, etc." added). \\
\hline 24 & Need to identify the cause of depressed feeling \\
\hline 25 & Unable to solve problems unassisted when depressed \\
\hline 26 & Unstable in mood \\
\hline 27 & Unable to switch moods unassisted \\
\hline 28 & Being short-tempered and/or showing unstable feelings \\
\hline 29 & Feeling irritated and/or restless \\
\hline 30 & Showing feelings of desperation \\
\hline 31 & Showing low self-esteem behaviors \\
\hline 32 & Pessimistic \\
\hline 33 & Expressionless \\
\hline 34 & Need to maintain motivation \\
\hline 35 & Decreasing motivation \\
\hline 36 & Lack of comprehension \\
\hline
\end{tabular}




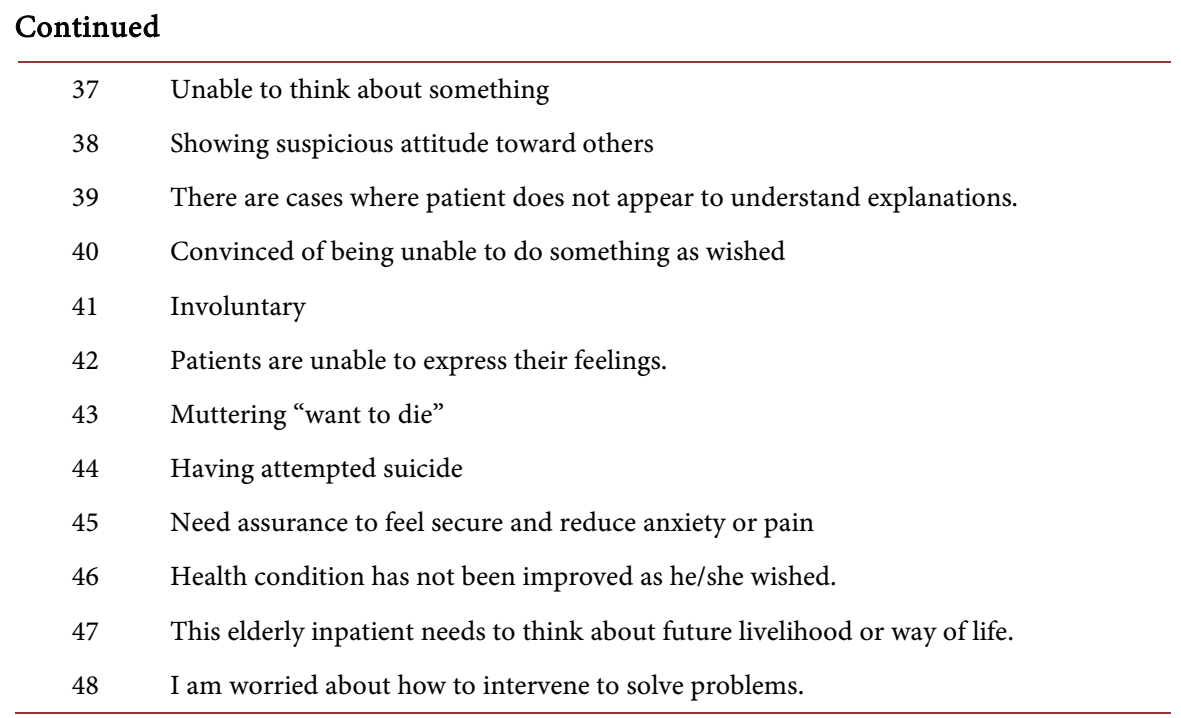

versions of the GDS15 and SDS. The reliability and validity of the GDS15 for Japanese elderly has been established by Yatomi [25], and that of the SDS by Niino [26]. To obtain uniform data without missing information, one researcher collected all the data of the interviews. On the day when the GDS15 and SDS were administered, the attending nurses were asked to fill in the NDE48 after completion of the day shift, here focusing on what they had observed that day. Next, between the following and the fifth day, different attending nurses of these participants were asked to fill in the NDE48. The elderly inpatients whose data were obtained from all of the GDS15, SDS, and NDE48 by the two different nurse groups were included as participants for the further analysis.

\subsubsection{Data Analysis}

Data were analyzed using the statistical analysis software R, ver 3.2.3.

(1) Validity

We determined the construct validity and criterion-related validity. The construct validity was examined with an oblique rotation (oblimin) of explanatory factor analysis, determining the factor loading, commonality, accumulated proportion, and factor correlation coefficient to confirm the factor structure of the NDE48. Oblique rotation was employed as it was assumed that there would be relations among the factors of the NDE48. The number of factors was confirmed using parallel analysis. For factor loadings of 0.40 or better, we determined this item as belonging to a factor [27], and employed the number of factors where the accumulated proportion was over 50\% [28]. Further, we excluded similar question items based on hierarchical and non-hierarchical cluster analyses. For the criterion-related validity, we examined the correlation coefficients of each of the total scores of the GDS15, SDS, and NDE48, and coefficients between 1.00 and 0.70 were considered as strongly correlated, between 0.70 and 0.40 as considerably correlated, and between 0.40 and 0.20 as slightly correlated [29].

(2) Reliability

For the reliability, we determined the internal consistency and inter-rater re- 
liability. For internal consistency, we looked at Cronbach's alpha coefficient of all question items and each factor as Powers \& Knapp (2011) have reported that: "the most common procedure for determining the internal consistency of a measuring instrument is Cronbach's alpha (p.90) [30].” The cutoff Cronbach's alpha coefficient level was set at 0.70 or higher [31], and inter-rater reliability was examined based on the correlation coefficient of the total score of NDE48 of each of the attending nurses. Standards for the correlation were the same as for the criterion-related validity.

(3) Ethical considerations

Inclusion of a hospital was made after the researcher had visited the hospital (all were hospitals with general wards in urban areas in Hokkaido) and had explained the study objectives and methods to the nursing directors in person. Hospitals that submitted a consent form after having obtained approval from their institutional review board were included as participating hospitals. After inclusion in the study the researcher explained the details of the study including the absence of any enforcement to participate, anonymity issues, and the confidentiality of the data to the elderly inpatients and attending nurses in writing, and obtained the consent to participate orally and in writing. Study data were kept in a secure locked location. Data input for the analysis were stored in a USB flash memory protected with a password. At the end of the study, all data to be destroyed after the research results have been published. It was decided that the data would be handled anonymously with unique codes by only the researchers, and when results of this study are presented at academic meetings or published in scientific journals, expressions that may identify participants and hospitals would not be used. The authors declare that they have no conflicts of interest. The protocol for the study was approved by the ethics committee of the university the authors belong to (No. 2014-098).

\section{Results}

\subsection{Demographic Characteristics of Study Participants and Attending Nurses}

Table 2 shows the demographic characteristics of the study participants and the attending nurses of the participants.

\subsubsection{Elderly Inpatients}

Thirty-two hospitals were invited to participate in the study, and of these seven hospitals agreed to cooperate, and introduced 58 elderly inpatients. Excluding four patients who did not express consent $(n=2)$ or whose data were not collected following the procedures detailed here $(\mathrm{n}=2)$; 54 patients $(93.1 \%)$ were included as participants of the study (35 female, 64.8\%; 19 male, 35.2\%). The mean age was 81.3 years old (SD, 7.56). None of the participants had a history of dementia or depression, one had unidentified complaints (1.9\%), and one suffered from dehydration (1.9\%). Twenty-five participants (46.3\%) had experienced losing a spouse, 28 (51.9\%) had no such experience, and for one this 
Table 2. Details of the participating elderly inpatients and attending nurses.

\begin{tabular}{|c|c|c|c|}
\hline & Item & Number & $\%$ \\
\hline Elderly inpatients & & \multicolumn{2}{|c|}{$\mathrm{n}=54$} \\
\hline Age & $81.3 \pm 7.51$ years & & \\
\hline \multirow{2}{*}{ Sex } & Female & 35 & 64.8 \\
\hline & Male & 19 & 35.2 \\
\hline \multirow{2}{*}{ Dementia } & No & 54 & 100.0 \\
\hline & Yes & 0 & 0.0 \\
\hline \multirow{2}{*}{ Depression } & No & 54 & 100.0 \\
\hline & Yes & 0 & 0.0 \\
\hline \multirow{2}{*}{$\begin{array}{l}\text { Unidentified } \\
\text { complaints }\end{array}$} & No & 53 & 98.1 \\
\hline & Yes & 1 & 1.9 \\
\hline \multirow{2}{*}{ Dehydration } & No & 53 & 98.1 \\
\hline & Yes & 1 & 1.9 \\
\hline \multirow{2}{*}{ Loss of spouse } & No & 28 & 51.9 \\
\hline & Yes & 25 & 46.3 \\
\hline \multirow{11}{*}{ Diseases } & $\begin{array}{l}\text { Diseases of the musculoskeletal system and connective } \\
\text { tissue }\end{array}$ & 15 & 27.8 \\
\hline & $\begin{array}{l}\text { Injury, poisoning, and certain other consequences of } \\
\text { external causes }\end{array}$ & 14 & 25.9 \\
\hline & Diseases of the respiratory system & 6 & 11.1 \\
\hline & Diseases of the circulatory system & 5 & 9.3 \\
\hline & Diseases of the genitourinary system & 4 & 7.4 \\
\hline & Neoplasms & 3 & 5.6 \\
\hline & Diseases of the nervous system & 2 & 3.7 \\
\hline & Endocrine, nutritional, and metabolic diseases & 2 & 3.7 \\
\hline & Diseases of the digestive system & 1 & 1.9 \\
\hline & Certain infectious and parasitic diseases & 1 & 1.9 \\
\hline & $\begin{array}{l}\text { Diseases of the blood and blood-forming organs and } \\
\text { certain disorders involving the immune mechanism }\end{array}$ & 1 & 1.9 \\
\hline \multicolumn{2}{|l|}{ Attending nurses } & \multicolumn{2}{|c|}{$\mathrm{n}=59$} \\
\hline \multirow{2}{*}{ Sex } & Female & 58 & 98.3 \\
\hline & Male & 1 & 1.7 \\
\hline $\begin{array}{l}\text { Length of nursing } \\
\text { experience }\end{array}$ & $12.6 \pm 8.65$ years & & \\
\hline \multirow{3}{*}{$\begin{array}{l}\text { Education to } \\
\text { obtain nurse } \\
\text { license }\end{array}$} & Vocational schools & 52 & 88.1 \\
\hline & Junior colleges & 3 & 5.1 \\
\hline & Universities & 4 & 6.8 \\
\hline \multirow{4}{*}{ Age } & $20 \mathrm{~s}$ & 12 & 20.3 \\
\hline & $30 \mathrm{~s}$ & 25 & 42.4 \\
\hline & $40 \mathrm{~s}$ & 14 & 23.7 \\
\hline & $50 \mathrm{~s}$ & 8 & 13.6 \\
\hline \multirow{6}{*}{ Assigned ward } & Rehabilitation at recovery & 6 & 10.2 \\
\hline & Internal medicine & 11 & 18.6 \\
\hline & Cardiovascular internal medicine & 5 & 8.5 \\
\hline & Digestive surgery & 14 & 23.7 \\
\hline & Orthopedic/internal medicine & 21 & 35.6 \\
\hline & Internal medicine/surgery & 2 & 3.4 \\
\hline
\end{tabular}


item is unknown (1.9\%). For the distribution of main complaints that led to the hospital admission according to the International Statistical Classification of Diseases and Related Health Problems (ICD-10, 2013) [32], 15 (27.8\%) were classified as diagnosed with diseases of the musculoskeletal system, and connective tissue, and 14 (25.9\%) with injury, poisoning, and other consequences of external causes. The mean score of the GDS15 was 5.77 (SD: 3.80), and 28 participants (51.8\%) scored 6 or higher, the cutoff point [33]. The mean score of the SDS was 40.83 (SD: 8.66), and 29 participants scored 40 or higher, which is classified as a higher than normal score [34].

\subsubsection{Attending Nurses}

The number of attending nurses was 59 (58 female, 98.3\%; 1 male, 1.7\%). The mean length of experience as a nurse was 12.58 years (SD: 8.65$)$. The nurse licenses held by $52(88.1 \%)$ of the participants were obtained at vocational nursing schools, by $3(5.1 \%)$ at junior colleges, and $4(6.8 \%)$ at universities.

\subsubsection{Data Collection from Attending Nurses}

The mean duration from admission to the first data collection from the attending nurses was 4.11 days (SD: 1.32), and that between the first and the second data collection was 1.24 days (SD: 0.77). The time required to complete NDE48 was 5.24 minutes (SD: 2.99) per patient.

\subsection{Validity}

\subsubsection{Construct Validity}

To determine the construct validity, we assigned 1 point to "Yes" responses, -1 point to "No", and 0 to "Unknown" responses, with the result that a higher total score implies a higher risk of a depressive state. Preliminarily assuming that there are three factors, we performed an oblique rotation (oblimin) for explanatory factor analysis and cluster analyses (hierarchical and non-hierarchical methods) with the N-GED48 scale. An Ansari-Bradley test with a nonparametric method for dispersion yielded $p=0.637$, showing no significant difference in dispersion, and we performed a factor analysis. Based on the assumed three factor classification, 25 of the 48 question items that appeared to measure similar conditions (eight items with low factor loadings and 17 items with factor loadings below 0.40) were excluded. For the remaining 23 items using parallel analysis, four factors were identified. Performing a factor analysis with these four factors, a further three items with factor loadings below 0.40 were excluded. From a factor analysis with the remaining 19 items, further two items with factor loadings below 0.40 were excluded. Examining the remaining 17 items using a parallel analysis, three factors were identified. This process was repeated, and one factor was found to have a factor loading below 0.40 and this was also excluded. Further, we performed a parallel analysis of the remaining 16 question items, and identified three factors. Table 3 shows the results of an oblique rotation (oblimin) of the explanatory factor analysis of the three factors of the remaining 16 items. As there were no items with factor loadings below 0.40 among these, and 
Table 3. Results of the factor analysis of the depressive state scale for elderly inpatients and Cronbach's $\alpha$ coefficients.

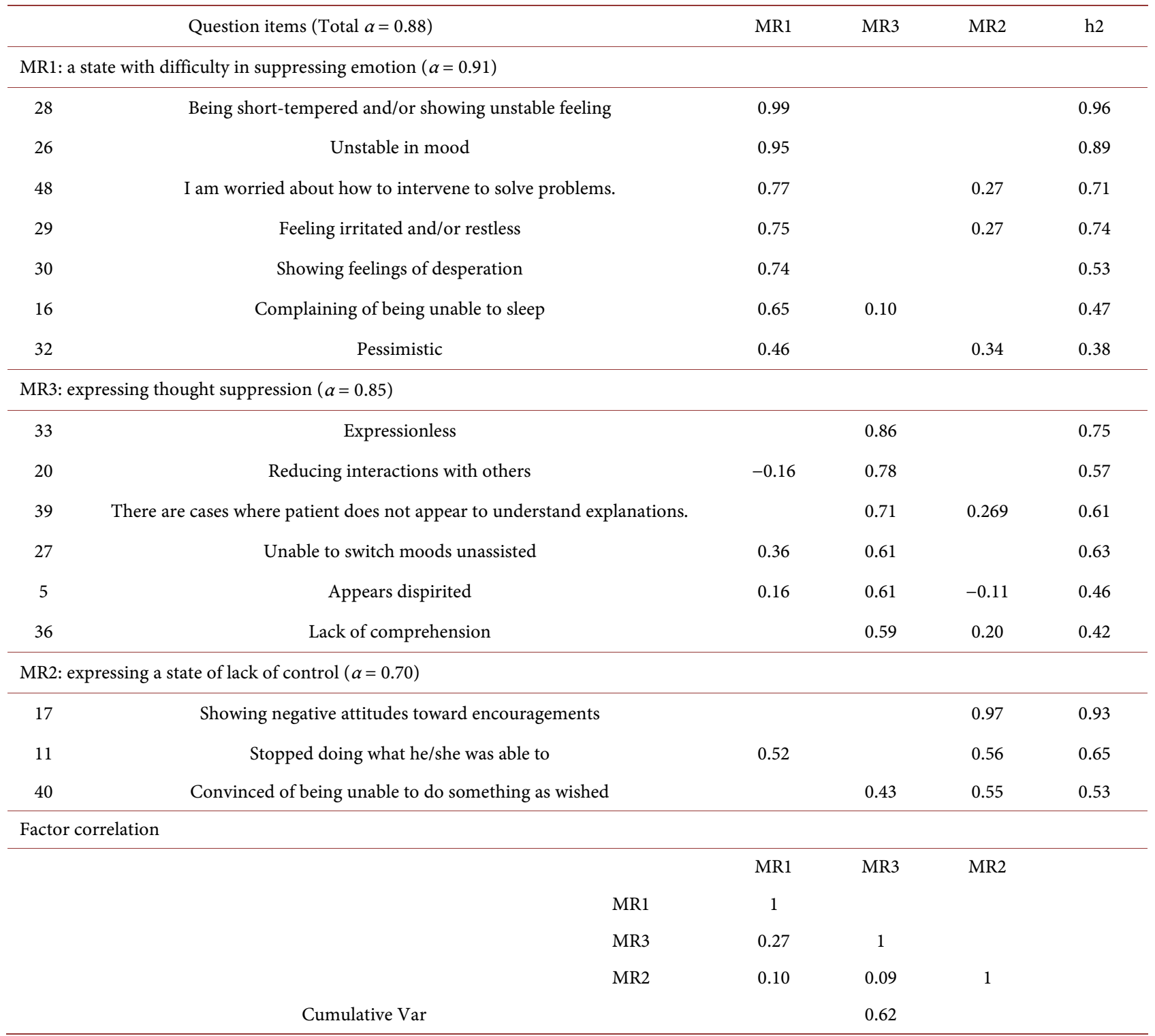

MR1: a state with difficulty in suppressing emotion $(\alpha=0.91)$, MR2: expressing a state of lack of control $(\alpha=0.70)$, MR3: expressing thought suppression $(\alpha$ $=0.85$ ).

the accumulated proportion was $62.0 \%$, it was decided that convergence had been reached. Finally, we determined to use these three factors and 16 question items and termed this the NDE16.The three factors were given the names "MR1: a state with difficulty in suppressing emotion", "MR2: expressing a state of lack of control", and "MR3: expressing thought suppression". The MR1 was comprised of question numbers, 16, 26, 28, 29, 30, 32 and 48; MR2 of 11, 17 and 40; and MR3 of 5, 20, 27, 33, 36 and 39. The correlation of these factors was from 0.09 to 0.27 .

\subsubsection{Criterion-Related Validity}

As a histogram showed no normal distribution of the total score of NDE16, we used the Spearman rank correlation to calculate correlation coefficients. The 
correlation coefficient (Spearman) between GDS15 and NDE16 was 0.41 ( $p=$ $0.00191)$, and between SDS and NDE16 was $0.30(p=0.02633)$.

\subsubsection{Reliability}

(1) Internal consistency

Table 3 shows Cronbach's $\alpha$ coefficients. Cronbach's $\alpha$ coefficient of the total score of NDE16 was 0.88 , and those of each factor were 0.91 (MR1), 0.70 (MR2), and 0.85 (MR3).

(2) Inter-rater reliability

The correlation coefficient (Spearman) between the data of the NDE16 collected from two different nurse groups was 0.57 ( $p=0.00005$ ).

\section{Discussion}

In this study we developed a scale for nurses to screen for the depressive state among elderly patients in general wards, and examined the reliability and validity of the scale statistically.

\subsection{Validity}

\subsubsection{Construct Validity}

Grove and Burns state that "Examination of construct validity determines whether the instrument actually measures the theoretical construct it purports measure (p. 217) [35]." Since the results of the explanatory factor analysis show that factor loadings were 0.40 or higher, and the accumulated proportion was $62.0 \%$, we determined that the validity of the NDE16 was verified. Further, as the correlation coefficients of the three factors were between 0.09 and 0.27 , little or no correlation among the three, the three factors are assumed to be independent or with very limited correlation.

For the symptoms characteristic to depressive states among the elderly, Igarashi reported that anxiety and feelings of frustration appear more strongly than feelings of depression if the person shows many somatic symptoms (indefinite complaints in particular) [36]. Put differently, depressive states among the elderly are unique in that they appear together with somatic symptoms, anxiety, and feelings of frustration. Question items included in the MR1 Factor of NDE16 include "28. Being short-tempered and/or showing unstable feelings", and "29. Feeling irritated and/or restless" from the initially developed draft scale (Table 1). These suggest depressive states among the elderly. Igarashi also noted general depressive states as states with mainly depressed feelings, thought suppressions and inhibition [36]. Factors, "MR2: expressing a state of lack of control" and "MR3: expressing thought suppression", in the final scale are typical symptoms of depressive states, and they represent thought suppression and inhibition.

The term depressive state in this study is defined as the level of depression where persons are depressed for a specific reason and are unable to cope with this by themselves, and which poses obstacles in daily life activities, including loss of appetite and insomnia, resulting in the need for nursing intervention. In 
other words, in this scale, three elements become emphasized, "being depressed", being "unable to cope with this by themselves" and being "in need of nursing intervention". As described above, we have determined that questions related to the MR1 Factor show characteristics of depressive states among the elderly, while those for the MR2 and MR3 Factors show the state of "being depressed" clearly because the characteristics of depressive states are shown in all developmental stages. These three factors all show that depressive states are equal in that they point to states that make these elderly "unable to cope with this by themselves" and "in need for nursing intervention", and therefore, we strongly feel that the scale developed in this study will actually measure depressive states in elderly inpatients.

\subsubsection{Criterion-Related Validity}

Powers and Knapp stated that "Criterion-related validity refers to the situation where the measures obtained are compared with some external criterion that has itself already been judged to be valid (a 'gold standard') (p. 197)" [30]. In the NDE16, the evaluating standards were examined by using GDS15 and SDS, of which the reliability and validity have been established. As a result, NDE16 is "fairly well correlated" with GDS15, and "slightly correlated" with SDS. This illustrates NDE16 is a scale more similar to GDS15 than SDS. Further, we have determined that NDE16 can measure depressive states because the correlation with GDS15 has been established. This strongly substantiates that the study aim to screen for depressive states among elderly inpatients has been achieved. Depressive states are also thought to be states where it takes a while from the time a person feels the symptoms of depression to the time others become able to observe the symptoms. The GDS15 and SDS are self-administered scales, but the scale developed here is based on observations by others. This difference may be the reason why there was no "strong correlation" between NDE16 and the other two scales.

\subsection{Reliability}

\subsubsection{Internal Consistency}

Powers and Knapp stated that "Internal consistency is a property of the reliability of a measuring instrument, and is concerned with the extent to which the parts of an instrument' hang together (p. 90)" [30]. Cronbach's $\alpha$ coefficient of the total score of NDE16 was 0.88 , and those of each factor were from 0.70 to 0.91 (MR1). As all the values were 0.70 or higher, we are confident to have determined the reliability of the question items and that each of the factors are supported as internally consist.

\subsubsection{Inter-Rater Reliability}

For inter-rater reliability, Polit and Beck (2012) states that it is "measurements by two or more observers or raters using the same instrument (repetition over persons) (p. 303) [37]." In the study here, the correlation coefficient (Spearman) of the total score of NDE16 obtained from different attending nurses was 0.57 ( $p$ 
$=0.00005)$, showing them to be "fairly well correlated", we have determined that the inter-rater reliability of the 16 question items is well supported.

\subsection{Usefulness of the Scale}

The scale developed here is unique in that it is completed by attending nurses, simple to fill out, and focuses specifically on depressive states among elderly inpatients. This scale is designed to enable nurses to identify early stage depressive states, as well as to contribute objective basic data to assist in interventions and medical examinations by physicians, rather than for the purpose of making a confirmed diagnosis. This scale can be used as an effective tool in countries and situations where there is a lack of knowledge of depression or where there are negative attitudes towards depression like in Japan as it is not necessarily completed by specialists who are knowledgeable of depression.

As the average time required to complete NDE48 was 5.24 minutes (SD: 2.99) for one patient, and because the total number of questions is only 16 , the scale is relatively easy to use. Further, because the simplified expressions in the wordings will enable a reliable determination regardless of the length of working experience of the nurses (or others) administering the scale, the scale does not impose any particular psychological burden on patients when the depressive states are evaluated. Nurses can use this scale easily and without collecting additional specific information.

It is reported that the incidence of depressive disorders among the elderly is high and there are often cases where no one notices the physical and mental changes experienced by the elderly [38]. Therefore, it is significant to have developed a scale exclusively for screening depressive states among elderly people. We think that the scale has the potential for use by a wide range of people including care givers and family members who are daily around the elderly. This is because the question items in NDE16 excluding number "48. I am worried about how to intervene to solve problems" were developed based on daily observations, and including number " 16 . Complaining of being unable to sleep".

\subsection{Limitations of the Present Study and Issues to be Addressed in Future Studies}

Limitations of the present study and issues to be addressed in future studies may be listed as follows:

- We feel assured that both the reliability and validity of the scale have been adequately established based on the statistical evidence obtained in this study. However, the number of participants $(n=54)$ is insufficient for 48 question items. This suggests a low stability for the method. Therefore, it is necessary to increase the number of participants to be able to effect improvements in the stability. Therefore, it is necessary to include more participants and examine the reliability and validity to ensure the stability of the NDE16 in the future studies.

- Taking account of the small number of questions (7 for MR1, 3 for MR2, and 
6 for MR3), we will work to improve the accuracy particularly paying attention to the balance among the three factors identified here.

- No participants with dementia were included in this study. However, in future studies the effectiveness of the scale in evaluating depressive states among elderly with dementia will be examined, as Byers and Yaffe (2011) state that "depressive symptoms often occur among patients with dementia" (P.324) [39].

\section{Conclusion}

This study set out to develop a scale for nurses to use to evaluate depressive states among elderly patients, and three factors represented by 16 question items were identified and presently comprise the scale. The three factors were named as "MR1: a state with difficulty in suppressing emotion", "MR2: expressing a state of lack of control", and "MR3: expressing thought suppression" to describe the areas highlighted by the three factors. The questions of the final scale that appear among the initially identified 48 questions in the draft scale were the following: MR1 comprised question numbers, 16, 26, 28, 29, 30, 32, and 48; MR2, 11, 17, and 40; and MR3, 5, 20, 27, 33, 36, and 39. Overall and taken together, the findings reported here suggest that the scale developed in this study is of potentially great use when nurses evaluate depressive states among elderly patients.

\section{Acknowledgements}

We wish to thank the nurses for their cooperation and participation in the study, and the nursing directors of the five participating hospitals for permitting us to conduct the survey. We are deeply grateful to Professor Kazumaru Wada, Graduate School of Health Sciences, Hirosaki University for the valuable supervision and support throughout our research. This research received no specific grant from any funding agency in the public, commercial, or not-for-profit sectors. The authors declare that they have no conflicts of interest.

\section{References}

[1] Cabinet Office of Japan (2015) Annual Report on the Aging Society. (In Japanese) http://www8.cao.go.jp/kourei/whitepaper/w-2016/html/gaiyou/s1_1.html

[2] Yamauchi, T. (1999) Critical Paths: What Can Be Learned about Them, What They Promise to Our Health Care, And How They Must Be Implemented. Journal of Oita University of Nursing and Health Sciences, 1, 11-19. (In Japanese)

[3] Organisation for Economic Co-Operation and Development (2016) Length of Hospital Stay. OECD, Tokyo. (In Japanese) https://data.oecd.org/healthcare/length-of-hospital-stay.htm

[4] Takeda, M. (2011) 6 Depressions: The Japan Geriatrics Society, Textbook of Geriatric Medicine. 3rd Edition, Medical View Co., Ltd., Tokyo, 81-82. (In Japanese)

[5] Takaoka, T. and Kidachi, R. (2016) Situations and Issues in Nursing Studies Related to Depression among the Elderly in Japan-Focusing on Search Results between 2003 and 2012. Journal of Japan Academy of Gerontological Nursing, 20, 68-75. (In Japanese) 
[6] Igarashi, Y. (2013) What Is a Depressive State? You Are a Good Physician! Identification and Diagnosis of "Depressive States"-Thinking: Oh? Perhaps? Strange? Japan Medical Journal, 26, 1-5. (In Japanese)

[7] Mizukami, K. (2009) Chapter 5, Depression and Other Illnesses-Dementia with Lewy Bodies and Senile Dementia. In: Mimura, M., Nakaaki, S. and Kocha, H., Eds., Handbook for Senile Dementia, Shindan To Chiryo Sha, Inc., Tokyo, 101-104. (In Japanese)

[8] Takahashi, Y. (2009) The Human Mind-New Revision of Depression in the Golden Age. Nippon Hyoronsha, Tokyo. (In Japanese)

[9] Hajjar, I., Yang, F., Sorond, F., Jones, R.N., Milberg, W., Cupples, L.A., et al. (2009) A Novel Aging Phenotype of Slow Gait, Impaired Executive Function, and Depressive Symptoms: Relationship to Blood Pressure and Other Cardiovascular Risks. The journals of gerontology. Series A, Biological Sciences and Medical Sciences, 64, 994-1001. https://doi.org/10.1093/gerona/glp075

[10] Moser, D.K., Dracup, K., Evangelista, L.S., Zambroski, C.H., Lennie, T.A., Chung, M.L., et al. (2010) Comparison of Prevalence of Symptoms of Depression, Anxiety, and Hostility in Elderly Patients with Heart Failure, Myocardial Infarction, and A Coronary Artery Bypass Graft. Heart \& Lung, 39, 378-385.

[11] Nabeta, H., Mizoguchi, Y., Matsushima, J., Imamura, J., Watanabe, I., Tateishi, T., et al. (2014) Association of Salivary Cortisol Levels and Later Depressive State in Elderly People Living in A Rural Community: A 3-Year Follow-up Study. Journal of Affective Disorders, 158, 85-89.

[12] Ogata, S., Hayashi, C., Sugiura, K. and Hayakawa, K. (2015) Associations between Depressive State and Impaired Higher-Level Functional Capacity in the Elderly with Long-Term Care Requirements. PLoS ONE, 10, e0127410. https://doi.org/10.1371/journal.pone.0127410

[13] Roesler, S.E., Sousa, A.R., Ferreira, L.B. and Peixoto, H.M. (2012) Prevalence and Factors Associated with Depression among Institutionalized Elderly Individuals: Nursing Care Support (in Portuguese). Revista da Escola de Enfermagem da USP, 46, 1387-1393.

[14] Yamazaki, S., Nakano, K., Saito, E. and Yasumura, S. (2012) Prediction of Functional Disability by Depressive State among Community-Dwelling Elderly in Japan: A Prospective Cohort Study. Geriatrics \& Gerontology International, 12, 680-687. https://doi.org/10.1111/j.1447-0594.2012.00841.x

[15] Takada, J., Meguro, K., Kurihara, Sato, Y., Chiba, Y. and Project Members (2014) Life Concerns of Elderly People Living at Home Determined as by Community General Support Center Staff: Implications for Organizing A More Effective Integrated Community Care System. Psychogeriatrics, 14, 188-195. https://doi.org/10.1111/psyg.12061

[16] Chen, C.S., Yang, M.S., Yang, M.J., Chang, S.J., Chueh, K.H., Su, Y.C., et al. (2008) Suicidal Thoughts among Elderly Taiwanese Aboriginal Women. International Journal of Geriatric Psychiatry, 23, 1001-1006. https://doi.org/10.1002/gps.2024

[17] Onishi, M. (2006) Factors Related to Social Support for The Elderly Living on Isolated Islands-Comparison of the Elderly Who Frequently Use Medical Services and Those Who Do Not. Nursing Journal of Kagawa University, 10, 25-32. (In Japanese)

[18] Yesavage, J.A. and Sheikh, J.I. (1986) 9/Geriatric Depression Scale (GDS) Recent Evidence and Development of a Shorter Version. Clinical Gerontologist, 5, 165-173. https://doi.org/10.1300/J018v05n01_09

[19] Zung, W.K. (1965) A Self-Rating Depression Scale. Archives of General Psychiatry, 
12, 63-70. https://doi.org/10.1001/archpsyc.1965.01720310065008

[20] Nakane, Y., Yoshioka, K. and Nakane, H. (2010) Chapter 3, Japan and Australia Comparative Joint Research on Mental Health. In: Aiming at Mental Barrier-Free Conditions for Depression and Schizophrenia among Japanese, Neisosyobo, Tokyo, 45-83. (In Japanese)

[21] Sakai, I. (2001) Development of a Check List to Screen Depressive States among Patients with Cerebrovascular Disorders. Quality Nursing, 7, 429-439. (In Japanese)

[22] Sakurai, H., Hosaka, T., Goto, A., Yamaguchi, I. and Ishida, Y. (2014) Development of a New Screening Tool for Delirium, Depression, and Dementia: Delirium, Depression, Dementia Screening Tool (3DST). Cancer Nursing, 19, 434-439.

[23] Prado-Jean, A., Couratier, P., Bénissan-Tevi, L.A., Nubukpo, P., Druet-Cabanac, M. and Clément, J.P. (2011) Development and Validation of an Instrument to Detect Depression in Nursing Homes. International Journal of Geriatric Psychiatry, 26, 853-859. https://doi.org/10.1002/gps.2613

[24] Benner, P. (2001) From Novice to Expert: Excellence and Power in Clinical Nursing Practice. Commemorative Edition, Prentice Hall, Upper Saddle River.

[25] Yatomi, N. (1994) The Factor Structure and Item Characteristics of The GDS (Geriatric Depression Scale) Short Version in a Japanese Elderly Sample. Japanese Journal of Gerontology, 16, 29-36.

[26] Niino, N. (1988) Reliability and Validity of Self-Rating Depression Scales for the Elderly. Japanese Journal of Public Health, 35, 201-203. (In Japanese)

[27] Tsushima, E. (2014) Chapter 9: Mechanisms of Factor Analysis. In: Medical Multivariate Data Analysis Learning with SPSS, Tokyo Tosho, Co., Ltd., Tokyo, 167-178. (In Japanese)

[28] Oshio, A. and Nishiguchi, T. (2012) Chapter 12: Procedures of Questionnaire Survey. In: Analysis Using SPSS, Nakanishiya Shuppan, Kyoto, 95-108. (In Japanese)

[29] Tsushima, E. (2014) Chapter 6: Correlation and Regression Analysis. In: Medical Data Analysis Learning with SPSS, Tokyo Tosho, Co., Ltd., Tokyo, 77-100. (In Japanese)

[30] Powers, B.A. and Knapp, T.R. (2011) Dictionary of Nursing Theory and Research. 4th Edition, Springer Publishing Company, New York.

[31] Yanai, H. and Ibe, T. (2013) Measurement in Nursing. In: Practical Issues in Questionnaire Surveys by Factor Analysis, Asakura Shoten, Tokyo, 132. (In Japanese)

[32] Ministry of Health, Labor and Welfare of Japan (2013) International Statistical Classification of Diseases and Related Health Problems (ICD-10, 2013). (In Japanese) http://www.mhlw.go.jp/toukei/sippei/

[33] Sugishita, M. and Asada, T. (2009) Development of A Geriatric Depression Scale. Short Version, GDS-S-J. Journal of Cognitive Neuroscience, 11, 87-90. (In Japanese)

[34] Fukuda, K. and Kobayashi, S. (2011) Manual for the Japanese Version of the Self-Rating Depression Scale. Expanded Edition, Sankyobo, Kyoto, 15. (In Japanese)

[35] Grove, K.S. and Burns, N. (2004) The Practice of Nursing Research: Conduct, Critique, \& Utilization. 5th Edition, Elsevier Saunders, St. Louis.

[36] Igarashi, Y. (2013) Chapter 2, How to Control Depressive States Arising from Physical Disorders, 13 "Depressive States" and Cerebrovascular Disorders and Parkinson's Disease, Focusing on the Elderly, for a Responsible Physician Identification and Diagnosis of "Depressive States". Japan Medical Journal, 26, 47-49. (In Japanese)

[37] Polit, D.F. and Beck, C.T. (2012) Nursing Research: Generating and Assessing Evi- 
dence for Nursing Practice. 10th International Edition, Lippincott Williams and Wilkins, Philadelphia.

[38] Tomimatsu, K. (2016) How to Support The Elderly Suffering from Depression for Practitioners in a Variety of Occupations. Pilae Press, Tokyo. (In Japanese)

[39] Byers, A.L. and Yaffe, K. (2011) Depression and Risk of Developing Dementia. Nature Reviews Neurology, 7, 323-331. https://doi.org/10.1038/nrneurol.2011.60

\section{Scientific Research Publishing}

Submit or recommend next manuscript to SCIRP and we will provide best service for you:

Accepting pre-submission inquiries through Email, Facebook, LinkedIn, Twitter, etc. A wide selection of journals (inclusive of 9 subjects, more than 200 journals)

Providing 24-hour high-quality service

User-friendly online submission system

Fair and swift peer-review system

Efficient typesetting and proofreading procedure

Display of the result of downloads and visits, as well as the number of cited articles Maximum dissemination of your research work

Submit your manuscript at: http://papersubmission.scirp.org/

Or contact ojn@scirp.org 